\title{
Analysis and Design of Distributed Antenna Aided Twin-Layer Femto-and Macro-Cell Networks Relying on Fractional Frequency-Reuse
}

\author{
Jie Zhang ${ }^{\dagger}$, Fan Jin*, Student Member, IEEE, Rong Zhang*, Member, IEEE, Guangjun $\mathrm{Li}^{\dagger}$, Member, IEEE and \\ Lajos Hanzo*, Fellow, IEEE \\ $\dagger$ School of Comm. and Info. Engineering, UESTC, China. \\ $*$ School of ECS., Univ. of Southampton, SO17 1BJ, UK. \\ Email: $\{j z 4, f j 1 \mathrm{~g} 10, \mathrm{rz}, \mathrm{lh}\} @$ @ecs.soton.ac.uk, http://www-mobile.ecs.soton.ac.uk
}

\begin{abstract}
Distributed Antenna Systems (DAS) and femtocells are capable of improving the attainable performance in the celledge area and in indoor residential areas, respectively. In order to achieve a high Spectral Efficiency (SE), both the Distributed Antenna Elements (DAEs) and Femtocell Base Stations (FBSs) may have to reuse the spectrum of the macrocellular network. As a result, the performance of both outdoor macrocell users and indoor femtocell users suffer from Co-Channel Interference (CCI). Hence in this paper, heterogenous cellular networks are investigated, where the DAS-aided macrocells and femtocells coexist within the same area. Both the outage probability and the spatially averaged throughput are derived and the network is optimised either for minimising the outage probability or for maximising the macrocell's throughput. Our analysis demonstrates that surprisingly, the Unity Frequency-Reuse (UFR) based macrocellular system can be optimised in isolation, without considering the impact of femtocells. We found that the macrocells relying on Fractional Frequency-Reuse (FFR) tend to be converted to several small cells, illuminated by the DAEs, when the density of femtocells becomes high.
\end{abstract}

\section{INTRODUCTION}

Heterogeneous Networks (HetNet) have become an essential part of the contemporary wireless landscape, since they are capable of achieving an improved coverage and power efficiency. A paradigm shift is taking place, indicating a trend, moving away from traditional high-power tower-mounted base stations (BS) towards heterogeneous low-power infrastructure elements [1]. This evolution relies on a diverse combination of techniques.

Most current literature on HetNets is focused on a simple conglomerate of heterogeneous network elements. By contrast, in this paper we consider two specific aspects of HetNets. Firstly, we consider a twin-layer network constituted by FemtoCells (FC) overlaid on Distributed Antenna System (DAS) aided MacroCells (MC) [2], [3] from a network-structural perspective. Secondly, we investigate three typical frequency planning arrangements of MCs, namely Unity Frequency Reuse (UFR), soft Fractional Frequency Reuse (soft-FFR) and

The financial support of the RC-UK under the auspices of the India-UK Advanced Technology Centre (IU-ATC) and that of the EU's Concerto project as well as that of the European Research Council's Advanced Fellow grant is gratefully acknowledged.

Copyright (c) 2013 IEEE. Personal use of this material is permitted. However, permission to use this material for any other purposes must be obtained from the IEEE by sending a request to pubs-permissions@ieee.org. hard Fractional Frequency Reuse (hard-FFR). Let us elaborate a little further on the rationale of these focal points.

The main limitation of cellular networks is the ubiquitous Co-Channel Interference (CCI), which is particularly damaging in the cell-edge area of systems employing the radical UFR, which the research community aspires to in the context of both the Universal Mobile Telecommunications System (UMTS) and in its Long Term Evolution (LTE). In the open literature, several solutions have been proposed to cope with CCI, such as time-domain techniques. The so-called, Almost blank subframe (ABSF) [4], [5] is one of the proposed timedomain techniques. Naturally, it is a straightforward practical solution for avoiding the strong CCI is to allocate orthogonal frequency bands within adjacent cells and reuse them in a certain pattern, leading to traditional Frequency Reuse (FR) [6]. A more sophisticated technique of exploiting the available frequency band is constituted by the Fractional Frequency Reuse (FFR) in its hard-FFR [7], [8] and soft-FFR [9], [10] variants, both of which improve the Area Spectral Efficiency (ASE) of classic FR, while maintaining a high Signal-toInterference-Ratio (SIR) in the cell-edge area. To elaborate a little further, the philosophy of FFR is that each cell is divided into a Cell-Centre Region (CCR) having access to the cell-centre's frequency band $F_{c}$ and the Cell-Edge Region (CER) having access to the cell-edge's frequency band $F_{e}$. The research of FFR system has been focused on determining the specific bandwidth of the frequency partitions [11]-[13], on the optimal resource management schemes conceived for static hard-FFR [14]-[16] and on the resource allocation problems of dynamic hard-FFR networks [9], [17].

In contrast to classic frequency planning invoked for combating the CCI, the DAS philosophy aims for providing an increased capacity, as its main design objective. Firstly, this is because the DAS may provide a shorter link between the Distributed Antenna Elements (DAEs) and the Mobile Station (MS) than that provided by the Macrocell Base Station (MBS), leading to a Reduced-Pathloss (RPL). Secondly, the DAS is capable of supporting several MSs within the same frequency band by exploiting their angular separation, leading to an angular multiplexing gain. Finally, when treating a DAS as a distributed Multi-User Multiple-Input and Multiple-Output (MU-MIMO) system, invoking advanced signal processing 
leads to both a macro-diversity gain and a multi-user gain 1 . As a result, we may naturally opt for a DAS in the CER in a FFR system, which is capable of further improving both the ASE and the achievable SIR of the CER.

Having considered the above-mentioned outdoor cellular performance issues, the coverage and capacity problem may even be more serious in indoor scenarios. In order to meet the traffic demands of indoor mobile users, FCs have been invoked as a cost-effective way of balancing the traffic of the entire cellular system. FCs may be overlaid onto MCs, forming a hierarchical twin-layer network structure [18]. Prior research on twin-layer cellular structures characterised both the UpLink (UL) and DownLink (DL) scenarios. Some of these contributions [19]-[21] derived the Outage Probability (OP) relying on the shared spectrum access policy by considering the coverage issues, while some authors [22], [23] considered assigning orthogonal spectral resources to the central MCs and to the FCs in order to eliminate their cross-layer interference. In addition to the above-mentioned centralised approaches, the authors of [24]-[26] proposed a distributed and self-organizing FC management scheme conceived for Orthogonal Frequency Division Multiple Access (OFDMA)-based cellular networks.

In order to support both the outdoor cellular users and indoor home users, operators may take advantage of the above three different approaches. Hence, we consider a DASaided twin-layer multi-cell OFDMA network and investigate, whether these promising techniques may be beneficially amalgamated. In particular, a range of important issues arise, such as the interference aspects of the twin-layer network, the employment of DASs, the power allocation of DASs as well as their frequency partitioning. Our contributions are as follows

- We invoke stochastic geometry [27], [28] for modelling the random distribution of FCs. The OP of both outdoor MC users and of home FC subscribers is derived in DAS-aided twin-layer networks. In contrast to [19]-[21] where the locations of the macro BSs are modeled as independent Spatial Poisson Point Processes (SPPP), we modelled the macrocell BSs as a grid model based circle of rings, as seen in Fig. 1. The grid based model is more popular and widely adopted for characterizing the multi-cell systems. Hence our model is more realistic comparing to [19]-[21].

- We study the impact of FCs on the existing DAS-aided MCs subject to different frequency planning strategies and optimise the DAS parameters, namely the power allocation between MBSs and DAEs as well as the FFRrelated frequency partitioning factor in order to either achieve the maximum MC throughput or to minimise the OP. This is achieved by improving the design approach of [11]-[14] upon amalgamating it with a Quality of Service (QoS) constrained design 2

The paper is organized as follows. In Section II, we describe our system model. Section III and Section IV elaborate on the

\footnotetext{
${ }^{1}$ However, due to the length limitation of our paper, we will not treat the most advanced applications of DASs in this paper.

${ }^{2}$ Given the length limitation of our paper, we consider the impact of FCs imposed on DAS-aided MCs, and will consider a holistically optimised solution in our future research.
}

analysis of DAS-aided twin-layer networks and then the corresponding optimisation problems are presented in Section V. Finally, our numerical results are provided in Section VI and our conclusions are offered in Section VII

\section{SySTEM MODEL}

\section{A. Topology Model}

The topology of our DAS-aided cellular network is illustrated in Fig. 11, where the over-sailing MCs are overlaid on top of the FCs and $N_{a}$ DAEs are employed in the CER of each MC, which are denoted by the triangles shown in Fig. 1 .

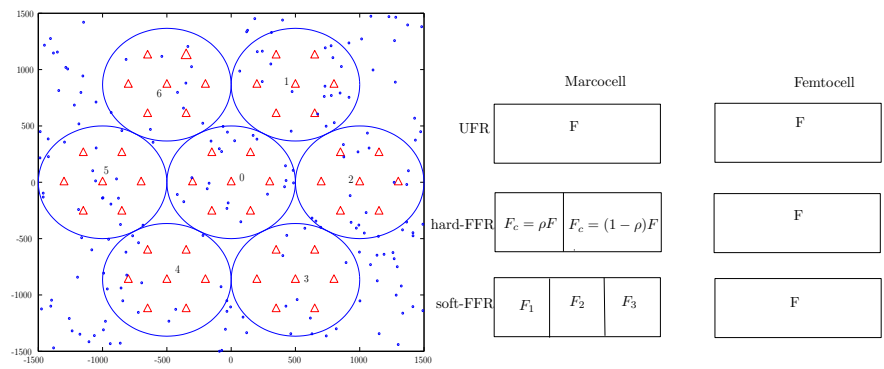

Fig. 1: The topology of the DAS-aided twin-layer cellular network and the frequency reuse strategies.

For analytical convenience, we model the MCs as circles with radii of $R_{m}$, where the central target $\mathrm{MBS} B_{0}^{m}$ is surrounded by six adjacent tier-one MBSs $B_{i}^{m}, i \in 1,2, \cdots, 6$. Within the $i$ th MBS $B_{i}^{m}, N_{a}=6$ DAEs denoted as $B_{i, j}^{a}, i \in$ $0,1, \cdots, 6 ; j \in, 1, \cdots, N_{a}$ are arranged symmetrically in the CER having radii of $r_{d}$, which are far from the MBS $B_{i}^{m}$. Observe furthermore from Fig. 1 that the FBSs $B_{n}^{f}, n \in \mathbb{N}$ are represented by the small circles within the area, which are randomly distributed, obeying a homogeneous Spatial Poisson Point Process (SPPP) according to an area-density of $\lambda$. Here, we denote the overall FBS set by $\Psi$, which is overlaid onto the DAS-aided MC network. The coverage area of each FBS is assumed to be a circle having a radius of $R_{f}$. In addition to the above BS configuration, we assume both the Outdoor MC Users (OMUs) and the Indoor FC Users (IFUs) to be independently and uniformly distributed in their coverage area. The MBSs and FBSs invoke the classic Round Robin (RR) scheduling strategy. Furthermore, the MBSs and DAEs will transmit their signals at their maximum power allowance of $P_{b}$ and $P_{a}$, respectively, while obeying the total transmit power constraint of $P_{b}+N_{a} P_{a}=P_{t}$, with $P_{t}$ denoting the total transmit power available for each MC. The FBSs will transmit their signals at their maximum power allowance of $P_{f}$.

In this paper, we consider three different FR strategies, namely UFR, hard-FFR and soft-FFR scenarios. For the UFR scenario, the total available bandwidth $F$ is used by both the MBS and by each DAE, where the OMUs are served either by the MBS or by the nearest DAE. In a hard-FFR scenario, the total available bandwidth $F$ is partitioned into four orthogonal frequency bands, namely $F_{c}, F_{1}, F_{2}$ and $F_{3}$, obeying $F=F_{c}+F_{1}+F_{2}+F_{3}$, where we assume that $F_{c}=\rho F$ and $F_{e}=F_{1}+F_{2}+F_{3}=(1-\rho) F$. Furthermore, $F_{c}$ represents the frequency bands available for the CCR, while 
$F_{i}, i \in 1,2,3$ represents the frequency bands available for the CER in one of the three adjacent MCs. When considering the soft-FFR strategy, the total available bandwidth $F$ is equally divided into three orthogonal frequency bands according to $F=F_{1}+F_{2}+F_{3}$, where one of the frequency bands $F_{i}, i \in 1,2,3$ is used in the CER, which is orthogonal to the neighbouring CER of the adjacent cells. On the other hand, the remaining two-thirds of the frequency band is reserved for the CCR.

\section{B. Channel Model}

In this paper, we assume that the DL channel is subject to uncorrelated Rayleigh fading obeying a unity average power constraint, as well as to wall-penetration loss and path-loss. For analytical simplicity, we considered a CCI-limited scenario and hence neglected the thermal noise in this paper. However, the consideration of the noise is straightforward. We let $d_{i}$ and $d_{i, j}$ denote the distance from a user to the $i$ th MBS $B_{i}^{m}$ and to the $j$ th DAE in the $i$ th $\mathrm{MC} B_{i, j}^{a}$, respectively, while $d_{f_{n}}$ represents the distance between the user and the $n$th FBS $B_{n}^{f}$. More specifically, the distance $d_{i}$ may be written as $d_{i}=\sqrt{r_{u}^{2}+r_{i}^{2}-2 r_{u} r_{i} \cos \left(\theta_{u}-\theta_{i}\right)}$, where $r_{u}$ and $\theta_{u}$ denote the radius and angle of the user, while $r_{i}$ and $\theta_{i}$ represent the radius and angle of the MBS $B_{i}^{m}$. Similarly, we have $d_{i, j}=\sqrt{r_{u}^{2}+r_{i, j}^{2}-2 r_{u} r_{i, j} \cos \left(\theta_{u}-\theta_{i, j}\right)}$ and $d_{f_{n}}=\sqrt{r_{u}^{2}+r_{f_{n}}^{2}-2 r_{u} r_{f_{n}} \cos \left(\theta_{u}-\theta_{f_{n}}\right)}$, where $r_{i, j}$ and $\theta_{i, j}$ denote the radius and angle of DAE $B_{i, j}^{a}$, while $r_{f_{n}}$ and $\theta_{f_{n}}$ represent the radius and angle of FBS $B_{n}^{f}$.

The DL propagation loss between a BS transmitter and a user is modelled as: $L=A d^{\alpha}$, where $A$ is a constant that depends on the carrier frequency $f_{c}, d$ denotes the length of this transceiver link, while $\alpha$ is the pathloss exponent. Furthermore, we denote the outdoor and indoor fading constant by $A_{O}$ and $A_{I}$, respectively. Finally, we denote the outdoor and indoor pathloss exponent by $\alpha_{1}$ and $\alpha_{2}$. The detailed channel model parameters are summarized in Table I]

\section{ANALYSIS OF DAS-AIDED TWIN-LAYER NETWORKS WITH UFR}

In this section, we will investigate the DL performance of DAS-aided twin-layer cellular networks associated with UFR, where the femtocells are close to the OMUs. The OP of both the OMUs and the IFUs will be derived, where an outage occurs when the instantaneous received SIR $\gamma$ of a transmission falls below a predefined threshold of $\gamma_{t h}$. Then, the spatially averaged throughput will be formulated.

\section{A. Outage probability of $O M U$}

The OMUs roaming in the CCR of the home MC $B_{0}^{m}$ suffer from CCI imposed by the DAEs $B_{i, j}^{a}$, by six tier-one MBSs $B_{i}^{m}, i \neq 0$, as well as by the interfering FBSs $B_{f_{n}}$. As a result, the associated SIR $\gamma_{u}$ of OMU $u$ in the CCR, which is located at a radius of $r_{u}$ and at an angle of $\theta_{u}$ from its home MBS
$B_{0}^{m}$, is given by:

$$
\gamma_{u}=\frac{\frac{P_{b}}{A_{O}} d_{0}^{-\alpha_{1}} h_{0}}{\sum_{i \neq 0} \frac{P_{b}}{A_{O}} d_{i}^{-\alpha_{1}} h_{i}+\sum_{i} \sum_{j} \frac{P_{a}}{A_{O}} d_{i, j}^{-\alpha_{1}} h_{i, j}+\sum_{n} \frac{P_{f}}{A_{O} W} d_{f_{n}}^{-\alpha_{1}} g_{n}},
$$

where $h_{i}, h_{i, j}$ and $g_{n}$ denote the negative exponentially decaying channel gain of unity mean with respect to (wrt) the MBS $B_{i}^{m}$, the DAE $B_{i, j}^{a}$ as well as the FBS $B_{n}^{f}$, respectively.

When the OMUs are located at the CER, they will be served by the DAE having the highest average received power, namely $k=\underset{j}{\operatorname{argmax}} \frac{P_{a}}{A_{O}} d_{0, j}^{-\alpha_{1}}$. Then the received SIR $\gamma_{u}$ of OMU having a radius of $r_{u}$ and an angle of $\theta_{u}$ from its MBS may be written as

$\gamma_{u}=\frac{\frac{P_{a}}{A_{O}} d_{0, j}^{-\alpha_{1}} h_{0, k}}{\sum_{i} \frac{P_{b}}{A_{O}} d_{i}^{-\alpha_{1}} h_{i}+\sum_{i \backslash 0} \sum_{j \backslash k} \frac{P_{a}}{A_{O}} d_{i, j}^{-\alpha_{1}} h_{i, j}+\sum_{n} \frac{P_{f}}{A_{O} W} d_{f_{n}}^{-\alpha_{1}} g_{n}}$.

In the DAS-aided twin-layer cellular network associated with UFR, the OP of OMUs is formulated in Lemma 1

Lemma 1: When an OMU $u$ located at a radius of $r_{u}$ and an angle of $\theta_{u}$ from its serving MBS $B_{0}^{m}$ in a DASaided twin-layer cellular network associated with UFR, the OP $\mathcal{O}_{u}\left(\gamma_{t h} \mid r_{u}, \theta_{u}\right)=P\left(\gamma_{u} \leq \gamma_{t h}\right)$ associated with the SIR threshold of $\gamma_{t h}$ may be written as

$\mathcal{O}_{u}\left(\gamma_{t h} \mid r_{u}, \theta_{u}\right)=1-e^{\left[-\pi \lambda C_{\alpha_{1}}\left(\frac{P_{f} \gamma_{t h}}{P_{b} d_{0}^{-\alpha}{ }^{-\alpha}}\right)^{\frac{2}{\alpha_{1}}}\right]} \mathcal{H}_{1} \quad u \in C C R$
$\mathcal{O}_{u}\left(\gamma_{t h} \mid r_{u}, \theta_{u}\right)=1-e^{\left[-\pi \lambda C_{\alpha_{1}}\left(\frac{P_{f} \gamma_{t h}}{P_{a} d_{0, k}^{-\alpha_{1} W}}\right)^{\frac{2}{\alpha_{1}}}\right]} \mathcal{H}_{2} \quad u \in C E R$,

where $\mathcal{H}_{1}=\prod_{i \neq 0}\left(1+\frac{d_{i}^{-\alpha_{1}} \gamma_{t h}}{d_{0}^{-\alpha_{1}}}\right)^{-1} \prod_{(i, j)} \prod_{1}\left(1+\frac{P_{a} d_{i, j}^{-\alpha_{1}} \gamma_{t h}}{P_{b} d_{0}^{-\alpha_{1}}}\right)^{-1}$, $\mathcal{H}_{2}=\prod_{i}\left(1+\frac{P_{b} d_{i}^{-\alpha_{1}} \gamma_{t h}}{P_{a} d_{0, k}^{-\alpha_{1}}}\right)^{-1} \prod_{(i, j) \backslash(0, k)}\left(1+\frac{d_{i, j}^{-\alpha_{1}} \gamma_{t h}}{d_{0, k}^{-\alpha_{1}}}\right)^{-1}$ and $C_{\alpha_{1}}=\int_{0}^{\infty} \frac{1}{1+s^{\frac{\alpha_{1}}{2}}} d s$.

Proof: See Appendix A

The OMUs are typically assumed to be independently and uniformly distributed within the coverage area. The probability density function of the OMU $u$ having a radius of $r$ and an angle of $\theta$ from its home MBS is given by: $p\left(r_{u}=r, \theta_{u}=\right.$ $\theta)=\frac{r}{\pi R_{m}^{2}}$. As a result, the spatially averaged OP $\mathcal{O}_{m}$ of OMUs may be written as

$$
\mathcal{O}_{m}\left(\gamma_{t h}\right)=\int_{0}^{R_{m}} \int_{0}^{2 \pi} \frac{r}{\pi R_{m}^{2}} \mathcal{O}_{u}\left(\gamma_{t h} \mid r, \theta\right) d r d \theta
$$

\section{B. Long-Term Spatially Averaged Macrocell Throughput}

We note that the Cumulative Distribution Function (CDF) of the $u$ th OMU's SIR $\gamma_{u}$ satisfies $\mathcal{F}_{\gamma_{u}}\left(\gamma_{t h} \mid r_{u}, \theta_{u}\right)=$ $\mathcal{O}_{u}\left(\gamma_{t h} \mid r_{u}, \theta_{u}\right)$, and the joint CDF of the received SIR $\gamma_{u}$ may be written as $\mathcal{F}_{\gamma_{u}}\left(\gamma_{t h}, r, \theta\right)=\mathcal{F}_{\gamma_{u}}\left(\gamma_{t h} \mid r_{u}, \theta_{u}\right) p\left(r_{u}=\right.$ $\left.r, \theta_{u}=\theta\right)$. As a result, the CDF of the spatially averaged SIR $\mathcal{F}_{\gamma_{m}}\left(\gamma_{t h}\right)$ of OMUs is given by $\mathcal{F}_{\gamma_{m}}\left(\gamma_{t h}\right)=$ $\int_{0}^{R_{m}} \int_{0}^{2 \pi} \frac{r}{\pi R_{m}^{2}} \mathcal{O}_{u}\left(\gamma_{t h} \mid r, \theta\right) d r d \theta$, which is the same as for the spatially averaged OP $\mathcal{O}_{m}$. 
The Probability Density Function (PDF) of the spatially averaged SIR for OMUs can be directly expressed by the derivative of the $\operatorname{CDF} \mathcal{F}_{\gamma_{m}}\left(\gamma_{t h}\right)$, which is written as $p\left(\gamma_{t h}\right)=$ $\frac{d \mathcal{F}_{\gamma_{m}}\left(\gamma_{t h}\right)}{d \gamma_{t h}}$. Hence, the achievable long-term spatially averaged throughput $T_{m}$ may be written using Shannon's classic formula as:

$$
\begin{aligned}
T_{m} & =\int_{0}^{\infty} \log _{2}\left(1+\gamma_{t h}\right) p\left(\gamma_{t h}\right) d \gamma_{t h} \\
& =\int_{0}^{\infty} \int_{0}^{R_{m}} \int_{0}^{2 \pi} \log _{2}\left(1+\gamma_{t h}\right) \frac{r}{\pi R_{m}^{2}} d \mathcal{O}_{u}\left(\gamma_{t h} \mid r, \theta\right) d r d \theta .
\end{aligned}
$$

\section{Outage Probability of IFU}

In this paper, we only investigate the performance of IFUs located at the cell-edge of their serving FBSs within the DAS-aided cellular network using the UFR strategy, which characterises the worst-case performance. Then, the IFUs are subjected to CCI emanating both from the MBSs / DAEs as well as from the other FBSs. As a result, the received SIR $\gamma_{u}$ of IFUs located at a distance $R_{f}$ from its serving FBS $B_{n}^{f}$ may be formulated as:

$$
\gamma_{u}=\frac{\frac{P_{f}}{A_{O}} R_{f}^{-\alpha_{2}} g_{n}}{\sum_{i} \frac{P_{b} d_{i}^{-\alpha_{1}}}{A_{O} W} h_{i}+\sum_{i} \sum_{j} \frac{P_{a} d_{i, j}^{-\alpha}}{A_{O} W} h_{i, j}+\sum_{j \neq n} \frac{P_{f} d_{f_{j}}^{-\alpha_{1}}}{A_{O} W^{2}} g_{j}} .
$$

The OP of the worst-case IFUs in a DAS-aided UFR scenario is formulated in Lemma 2

Lemma 2: When the IFUs are located at the cell-edge of its serving FBS $B_{n}^{f}$, which is far from the anchor MBS $B_{0}^{m}$ with a radius of $r_{f_{n}}$ and an angle of $\theta_{f_{n}}$, the OP $\mathcal{O}_{u}\left(\gamma_{t h} \mid r_{f_{n}}, \theta_{f_{n}}\right)=$ $P\left(\gamma_{u} \leq \gamma_{t h}\right)$ may be written as:

$$
\mathcal{O}_{u}\left(\gamma_{t h} \mid r_{f_{n}}, \theta_{f_{n}}\right)=1-e^{\left[-\pi \lambda C_{\alpha_{1}}\left(\frac{\gamma_{t h}}{W^{2} R_{f}^{-\alpha_{2}}}\right)^{\frac{2}{\alpha_{1}}}\right]} \mathcal{H}_{3},
$$

where $\mathcal{H}_{3}=\prod_{i}\left(1+\frac{P_{b} \gamma_{t h} d_{i}^{-\alpha_{1}}}{P_{f} W R_{f}^{-\alpha_{2}}}\right)^{-1} \prod_{i} \prod_{j}\left(1+\frac{P_{a} \gamma_{t h} d_{i, j}^{-\alpha_{1}}}{P_{f} W R_{f}^{-\alpha_{2}}}\right)^{-1}$.

$$
\text { Proof: See Appendix B }
$$

In the literature, the FBSs are also typically assumed to be independently and uniformly distributed within the cellular network, hence the probability density function of the FBS being located at a radius of $r$ and at an angle of $\theta$ is given by $p\left(r_{f_{n}}=r, \theta_{f_{n}}=\theta\right)=\frac{r}{\pi R_{m}^{2}}$. The spatially averaged OP $\mathcal{O}_{f}$ of IFUs, which are in the CER of their serving FBS may be written as

$$
\mathcal{O}_{f}\left(\gamma_{t h}\right)=\int_{0}^{R_{m}} \int_{0}^{2 \pi} \frac{r}{\pi R_{m}^{2}} \mathcal{O}_{u}\left(\gamma_{t h} \mid r, \theta\right) d r d \theta .
$$

\section{Long-Term Spatially Averaged Femtocell Worst-Case Throughput}

The CDF of the spatially averaged worst-case $\operatorname{SIR} \mathcal{F}_{\gamma_{f}}\left(\gamma_{t h}\right)$, is given by

$$
\mathcal{F}_{\gamma_{f}}\left(\gamma_{t h}\right)=\int_{0}^{R_{m}} \int_{0}^{2 \pi} \frac{r}{\pi R_{m}^{2}} \mathcal{O}_{u}\left(\gamma_{t h} \mid r, \theta\right) d r d \theta .
$$

Again, the PDF of the spatially averaged SIR for the worstcase IFUs is directly given by the derivative of the CDF $\mathcal{F}_{\gamma_{f}}\left(\gamma_{t h}\right)$, which is written as $p\left(\gamma_{t h}\right)=\frac{d \mathcal{F}_{\gamma_{f}}\left(\gamma_{t h}\right)}{d \gamma_{t h}}$. Then, the achievable long-term spatially averaged FC throughput $T_{f}$ may be written as

$$
\begin{aligned}
T_{f} & =\int_{0}^{\infty} \log _{2}\left(1+\gamma_{t h}\right) p\left(\gamma_{t h}\right) d \gamma_{t h} \\
& =\int_{0}^{\infty} \int_{0}^{R_{m}} \int_{0}^{2 \pi} \log _{2}\left(1+\gamma_{t h}\right) \frac{r}{\pi R_{m}^{2}} d \mathcal{O}_{u}\left(\gamma_{t h} \mid r, \theta\right) d r d \theta .
\end{aligned}
$$

\section{ANalysis of DAS-AIDED Twin-LAYER NETWORKS WITH FFR}

In an FFR scenario, the total frequency band $F$ is divided into the orthogonal frequency bands of $F_{c}$ and $F_{e}$, which are reserved for the CCR and the CER, respectively.

\section{A. DAS-aided DL Transmission with hard-FFR}

1) Outage probability of OMU: In a hard-FFR scenario, the OMUs roaming in the CCR receive no CCI from the DAEs any more, hence the received SIR $\gamma_{u}$ of the $u$ th OMU having a radius of $r_{u}$ and an angle of $\theta_{u}$ from its home MBS may be expressed as

$$
\gamma_{u}=\frac{\frac{P_{b}}{A_{0}} d_{0}^{-\alpha_{1}} h_{0}}{\sum_{i \neq 0} \frac{P_{b}}{A_{0}} d_{i}^{-\alpha_{1}} h_{i}+\sum_{n} \frac{P_{f}}{A_{0} W} d_{f_{n}}^{-\alpha_{1}} g_{n}} .
$$

When the OMU is far from the home MBS and located in the CER, it will be served by the DAE having the highest received power. In other words, the OMU will be supportted by the DAE $B_{0, k}^{a}$ with $k=\underset{j}{\operatorname{argmax}} \frac{P_{a}}{A_{0}} d_{0, j}^{-\alpha_{1}}$. Then, the OMU may suffer from strong CCI imposed both by other DAEs within the same MC as well as by the interfering FBSs, and the corresponding SIR $\gamma_{u}$ may be written as

$$
\gamma_{u}=\frac{\frac{P_{a}}{A_{0}} d_{0, k}^{-\alpha_{1}} h_{0, k}}{\sum_{j \neq k} \frac{P_{a}}{A_{0}} d_{0, j}^{-\alpha_{1}} h_{0, j}+\sum_{n} \frac{P_{f}}{A_{0} W} d_{f_{n}}^{-\alpha_{1}} g_{n}} .
$$

Following a similar derivation to that of Lemma1, the OP of OMUs operating in a hard-FFR scenario is given by Lemma 3 .

Lemma 3: In DAS-aided twin-layer cellular networks associated with a hard-FFR frequency reuse pattern, the OP $\mathcal{O}_{u}\left(\gamma_{t h} \mid r_{u}, \theta_{u}\right)$ of OMUs located at a radius of $r_{u}$ and an angle of $\theta_{u}$ from their home MBS may be written as

$$
\begin{aligned}
& \mathcal{O}_{u}\left(\gamma_{t h} \mid r_{u}, \theta_{u}\right)=1-e^{\left[-\pi \lambda C_{\alpha_{1}}\left(\frac{P_{f} \gamma_{t h}}{P_{b} W d_{0}^{-\alpha_{1}}}\right)^{\frac{2}{\alpha_{1}}}\right]} \mathcal{H}_{4} u \in C C R, \\
& \mathcal{O}_{u}\left(\gamma_{t h} \mid r_{u}, \theta_{u}\right)=1-e^{\left[-\pi \lambda C_{\alpha_{1}}\left(\frac{P_{f} \gamma_{t h}}{P_{a} W d_{0, k}^{-\alpha_{1}}}\right)^{\frac{2}{\alpha_{1}}}\right]} \mathcal{H}_{5} \quad u \in C E R, .
\end{aligned}
$$

where we have $\mathcal{H}_{4}=\prod_{i \neq 0}\left(1+\frac{d_{i}^{-\alpha_{1}} \gamma_{t h}}{d_{0}^{-\alpha_{1}}}\right)^{-1}$ and $\mathcal{H}_{5}=\prod_{i \neq k}(1+$ $\left.\frac{d_{0, j}^{-\alpha_{1}} \gamma_{t h}}{d_{0, k}^{-\alpha_{1}}}\right)^{-1}$. 
By exploiting the assumption that the OMUs are independently and uniformly distributed in the coverage area, the spatially averaged OP $\mathcal{O}_{m}\left(\gamma_{t h}\right)$ of OMUs may be written as

$$
\mathcal{O}_{m}\left(\gamma_{t h}\right)=\int_{0}^{R_{m}} \int_{0}^{2 \pi} \frac{r}{\pi R_{m}^{2}} \mathcal{O}_{u}\left(\gamma_{t h} \mid r, \theta\right) d r d \theta .
$$

2) Long-Term Spatially Averaged Macrocell Throughput: Similar to the UFR scenario, the CDF of the spatially averaged SIR $\mathcal{F}_{\gamma_{m}}\left(\gamma_{t h}\right)$ and the long-term spatially averaged throughput $T_{m}$ may be written as

$$
\begin{aligned}
& \mathcal{F}_{\gamma_{m}}\left(\gamma_{t h}\right)=\int_{0}^{R_{m}} \int_{0}^{2 \pi} \frac{r}{\pi R_{m}^{2}} \mathcal{O}_{u}\left(\gamma_{t h} \mid r, \theta\right) d r d \theta \\
& T_{m}=\int_{0}^{\infty} \int_{0}^{R_{m}} \int_{0}^{2 \pi} \log _{2}\left(1+\gamma_{t h}\right) \frac{r}{\pi R_{m}^{2}} d \mathcal{O}_{u}\left(\gamma_{t h} \mid r, \theta\right) d r d \theta,
\end{aligned}
$$

where $\mathcal{O}_{m}\left(\gamma_{t h}\right)$ denotes the spatially averaged MC OP and $\mathcal{O}_{u}\left(\gamma_{t h} \mid r, \theta\right)$ is the OP of the $u$ th OMU, which is given by Eq. (11) and Lemma 3, respectively.

\section{B. DAS-aided DL Transmission with soft-FFR}

1) Outage probability of $O M U$ : In a soft-FFR frequency reuse pattern, the total frequency band $F$ is equally divided into three orthogonal frequency bands according to $F=F 1+$ $F 2+F 3$, where one of them is reserved for the CER and the remaining two are used in the CCR. Hence, the OMUs roaming in the CCR suffer from no CCI due to the DAEs within the same MC any more in a soft-FFR scenario, while both the MBSs and DAEs of tier-one MCs impose CCI on the CCR's OMUs. As a result, the OP of OMUs in the CCR becomes worse than that of the hard-FFR scenario and the received SIR $\gamma_{u}$ of the OMU $u$ at the radius of $r_{u}$ and angle of $\theta_{u}$, but far from its anchor MBS may be expressed as

$$
\gamma_{u}=\frac{\frac{P_{b}}{A_{0}} d_{0}^{-\alpha_{1}} h_{0}}{\sum_{i \neq 0} \frac{P_{b} d_{0}^{-\alpha_{1}}}{A_{0}} h_{i}+\sum_{i \neq 0} \sum_{j} \frac{P_{a} d_{i, j}^{-\alpha_{1}}}{A_{0}} h_{i, j}+\sum_{n} \frac{P_{f} d_{f_{n}}^{-\alpha_{1}}}{A_{0} W} g_{n}} .
$$

When the OMU is roaming in the CER, no CCI will be imposed by its anchor MBS, while its received SIR $\gamma_{u}$ depends on the CCI emanating both from the tier-one MBSs as well as from the interfering FBSs using the same frequency band. The received SIR may be written as:

$$
\gamma_{u}=\frac{\frac{P_{a}}{A_{0}} d_{0, k}^{-\alpha_{1}} h_{0, k}}{\sum_{i \neq 0} \frac{P_{b}}{A_{0}} d_{i}^{-\alpha_{1}} h_{i}+\sum_{n} \frac{P_{f}}{A_{0} W} d_{f_{n}}^{-\alpha_{1}} g_{n}} .
$$

Lemma 4: In DAS-aided twin-layer cellular networks associated with soft-FFR, the OP of OMU $\mathcal{O}_{u}\left(\gamma_{t h} \mid r_{u}, \theta_{u}\right)$ may be written as

$$
\begin{aligned}
& \mathcal{O}_{u}\left(\gamma_{t h} \mid r_{u}, \theta_{u}\right)=1-e^{\left[-\pi \lambda C_{\alpha_{1}}\left(\frac{P_{f} \gamma_{t h}}{P_{b} d_{0}^{-\alpha_{1}} W}\right)^{\frac{2}{\alpha_{1}}}\right]} \mathcal{H}_{6} \quad u \in C C R, \\
& \mathcal{O}_{u}\left(\gamma_{t h} \mid r_{u}, \theta_{u}\right)=1-e^{\left[-\pi \lambda C_{\alpha_{1}}\left(\frac{P_{f} \gamma_{t h}}{P_{b} d_{0, k}^{-\alpha_{1} W}}\right)^{\frac{2}{\alpha_{1}}}\right]} \mathcal{H}_{7} u \in C E R,
\end{aligned}
$$

we have $\mathcal{H}_{6}=\prod_{i \neq 0}\left(1+\frac{d_{i}^{-\alpha_{1}} \gamma_{t h}}{d_{0}^{-\alpha_{1}}}\right)^{-1} \prod_{i \neq 0} \prod_{j}\left(1+\frac{P_{a} d_{i, j}^{-\alpha_{1}} \gamma_{t h}}{P_{b} d_{0}^{-\alpha_{1}}}\right)^{-1}$ and $\mathcal{H}_{7}=\prod_{i \neq 0}\left(1+\frac{P_{b} d_{i}^{-\alpha_{1}} \gamma_{t h}}{P_{a} d_{0, k}^{-\alpha_{1}}}\right)^{-1}$.

Similarly, the spatially averaged OP $\mathcal{O}_{m}\left(\gamma_{t h}\right)$ of OMUs may be written as

$$
\mathcal{O}_{m}\left(\gamma_{t h}\right)=\int_{0}^{R_{m}} \int_{0}^{2 \pi} \frac{r}{\pi R_{m}^{2}} \mathcal{O}_{u}\left(\gamma_{t h} \mid r, \theta\right) d r d \theta .
$$

2) Long-Term Spatially Averaged Macrocell Throughput: According to the assumption of having independently and uniformly distributed OMUs in the MC, the CDF of the spatially averaged SIR $\mathcal{F}_{\gamma_{m}}\left(\gamma_{t h}\right)$ and the long-term spatially averaged throughput $T_{m}$ may be written as

$$
\begin{aligned}
& \mathcal{F}_{\gamma_{m}}\left(\gamma_{t h}\right)=\int_{0}^{R_{m}} \int_{0}^{2 \pi} \frac{r}{\pi R_{m}^{2}} \mathcal{O}_{u}\left(\gamma_{t h} \mid r, \theta\right) d r d \theta \\
& T_{m}=\int_{0}^{\infty} \int_{0}^{R_{m}} \int_{0}^{2 \pi} \log _{2}\left(1+\gamma_{t h}\right) \frac{r}{\pi R_{m}^{2}} d \mathcal{O}_{u}\left(\gamma_{t h} \mid r, \theta\right) d r d \theta
\end{aligned}
$$

where, again, the spatially averaged OP $\mathcal{O}_{m}\left(\gamma_{t h}\right)$ and the OP $\mathcal{O}_{u}\left(\gamma_{t h} \mid r, \theta\right)$ of the $u$ th OMU at the radius of $r_{u}=r$ and angle of $\theta_{u}=\theta$ is given by Eq. (16) and by Lemma 4 respectively.

\section{Optimal Design of DAS-Aided Twin-Layer NETWORK}

We will focus our attention on the optimal design of the DAS-aided twin-layer system using UFR, hard-FFR and softFFR frequency reuse pattern. Specifically, we will optimise both the normalised radii $r_{d}$ of the DAS and the power allocation factor $\mu=\frac{P_{a}}{P_{b}}$ for UFR and soft-FFR scenarios. Similarly, we will also optimise the normalised radii $r_{d}$ of the DAS, the power allocation factor $\mu=\frac{P_{a}}{P_{b}}$ as well as the spectrum partitioning factor $\rho$ of the hard-FFR scenario. The appropriate choice of these parameters is expected to achieve the maximum total long-term spatially averaged MC throughput per subchannel $T_{m}$ or, alternatively to minimise the spatially averaged OP $\mathcal{O}_{m}\left(\gamma_{t h}\right)$.

\section{A. OP-based Design}

In this paper, we present an OP-based design approach with the aim of minimising the spatially averaged OP $\mathcal{O}_{m}\left(\gamma_{t h}\right)$, where we stipulate the QoS requirement $\eta$, guaranteeing that the long-term spatially averaged CER throughput is at least a fraction $\eta$ of the CCR throughput. Hence, the system parameters may be adjusted for trading the data-rates of the CCR MC users against those of the CER MC users. Given the predefined SIR threshold $\gamma_{t h}$, the optimisation problem using this OP-based approach may be formulated as

$$
\begin{array}{ll}
\min & \mathcal{O}_{m}\left(\gamma_{t h}, r_{d}, \mu\right)=\int_{0}^{R_{m}} \int_{0}^{2 \pi} \frac{r}{\pi R_{m}^{2}} \mathcal{O}_{m}\left(\gamma_{t h} \mid r, \theta\right) d r d \theta \\
\text { s.t. } & \frac{\rho_{o} N_{a} T_{m_{2}}\left(r_{d}, \mu\right)}{\rho_{i} T_{m_{1}}\left(r_{d}, \mu\right)} \geq \eta \\
& P_{b}+N_{a} P_{a}=P_{t}
\end{array}
$$


where $T_{m_{1}}$ and $T_{m_{2}}$ denote the spatially averaged throughput within the CCR and the CER of the MC, respectively. Furthermore, $\rho_{o}$ and $\rho_{i}$ denote the normalised available frequency bands within the CER and within the CCR, respectively. More specifically, the normalised frequency bands available satisfy $\rho_{o}=\rho_{i}=1$ within the UFR scenario and $\rho_{o}=\frac{1}{3}$ and $\rho_{i}=\frac{2}{3}$ within the soft-FFR scenario, while $\rho_{o}$ and $\rho_{i}$ are the function of the frequency partitioning factor $\rho$ of $\rho_{o}=\frac{1-\rho}{3}$ and of $\rho_{i}=\rho$ within the hard-FFR scenario.

\section{B. Throughput-based Design}

In addition to the OP-based design, we also present a throughput-based design approach conceived for maximising the average MC throughput of UFR, hard-FFR and of soft-FFR scenarios, where the above-mentioned QoS requirement $\eta$ is also employed. When a predefined SIR threshold $\gamma_{t h}$ is given, the throughput-based optimisation problem may be formulated as

$$
\begin{array}{ll}
\max & T_{m}\left(r_{d}, \mu, \rho\right)=\rho_{i} T_{m_{1}}\left(r_{d}, \mu\right)+\rho_{o} N_{a} T_{m_{2}}\left(r_{d}, \mu\right) \\
\text { s.t. } & \frac{\rho_{o} N_{a} T_{m_{2}}\left(r_{d}, \mu\right)}{\rho_{i} T_{m_{1}}\left(r_{d}, \mu\right)} \geq \eta \\
& P_{b}+N_{a} P_{a}=P_{t} .
\end{array}
$$

Remark 1: Note that the constraint imposed by the inequality $\frac{\rho_{o} N_{a} T_{m_{2}}\left(r_{d}, \mu\right)}{\rho_{i} T_{m_{1}}\left(r_{d}, \mu\right)} \geq \eta$ is a linear one, hence the problem can be solved by the method of Lagrange multipliers. The optimum frequency partitioning factor $\rho^{*}$ of the hard-FFR scenario is determined by the extremal points of the inequality, which may be written as $\rho^{*}=\frac{N_{a} T_{m_{2}}\left(r_{d}, \mu\right)}{N_{a} T_{m_{2}}\left(r_{d}, \mu\right)+3 \eta T_{m_{1}}\left(r_{d}, \mu\right)}$.

\section{Optimisation tool}

Since no closed-form equations have been found for the OP $\mathcal{O}_{m}\left(\gamma_{t h}, r_{d}, \mu\right)$ and for the MC throughput $T_{m_{i}}\left(r_{d}, \mu\right), i \in$ 1,2 , we solve the optimisation problems of Eq. (19) and Eq. (20) using the classic GA optimisation method [29], where $T_{m_{1}}\left(r_{d}, \mu\right)$ and $T_{m_{2}}\left(r_{d}, \mu\right)$ will be numerically evaluated with the aid of Matlab tools due to the fact that the three-fold integration is extremely time-consuming. The GA constitutes an attractive global heuristic optimisation tool for search problems, which mimics the process of natural evolution. A random population $P$ is created first, and the fitness of each individual candidate-solution is evaluated. Then a number of high-fitness individuals are selected from the current generation of the population in order to create off-springs with the aid of using the standard crossover and mutation operations. The newly formed generation of the population is then used in the next iteration of the algorithm, hence the GA process leads to the gradual evolution of the population to better solutions of the optimisation problem. The optimisation process using the classic GA is briefly summarised in Appendix C

\section{Performance Evaluation}

In this section, we will evaluate the performance of DASaided twin-layer cellular networks using our UFR, hard-FFR and soft-FFR strategies. The CDF of the received SIR of both the OMUs and IFUs is verified first, which is followed by the results of our optimal design. Our system parameters are summarised in Table 1 .

TABLE I: Notations and System parameters

\begin{tabular}{|l|l|r|}
\hline Symbol & Description & Value \\
\hline$f_{c}$ & Carrier frequency & $2 \mathrm{GHz}$ \\
\hline$D$ & Inter-Cell Distance & $1000 \mathrm{~m}$ \\
\hline$R_{f}$ & Radius of the Femtocell & $20 \mathrm{~m}$ \\
\hline$A_{O, d B}$ & Fixed outdoor pathloss & $33.26 \log _{10}\left(f_{c}\right)-79.86 \mathrm{~dB}$ \\
\hline$A_{I, d B}$ & Fixed indoor pathloss & $37 \mathrm{~dB}$ \\
\hline$\alpha_{1}$ & Outdoor pathloss exponent & 4 \\
\hline$\alpha_{2}$ & Indoor pathloss exponent & 2 \\
\hline$W_{d B}$ & Wall penetration loss & $5 \mathrm{~dB}$ \\
\hline$P_{m, d B}$ & MBS transmit power & $46 \mathrm{dBm}$ \\
\hline$P_{f, d B}$ & FBS transmit power & $13 \mathrm{dBm}$ \\
\hline
\end{tabular}

\section{A. CDF of Spatially Received SIR}

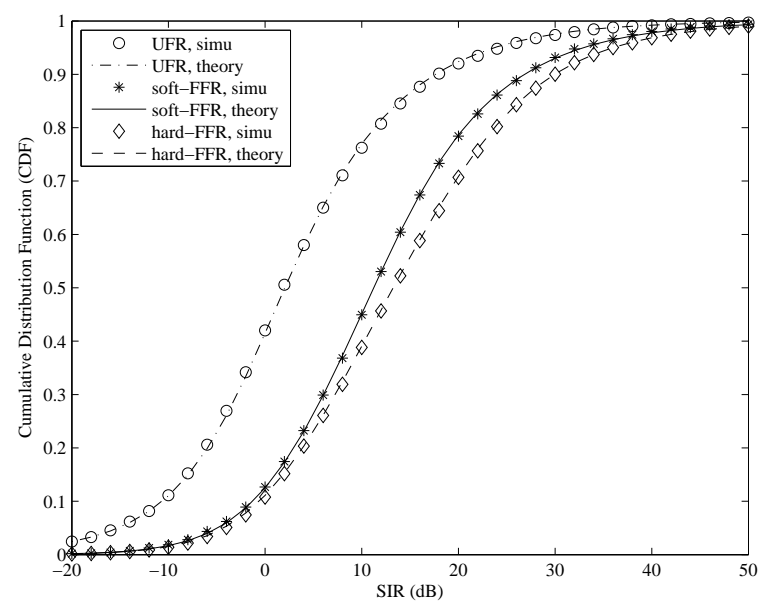

Fig. 2: The CDF of the received SIR in the absence of femtocells.

Fig. 2 and Fig. 3 illustrate the CDF of the spatially averaged received SIR $\gamma$ within DAS-aided twin-layer cellular networks, where the FBSs are randomly distributed obeying a homogeneous SPPP according to an area-density of $\lambda=\frac{N_{f}}{\pi R_{m}^{2}}$ with $N_{f}$ denoting the averaged number of FCs within each MC. Observe from Fig. 2 that our theoretical results of the received SIR recorded for OMUs quite accurately match the simulation results, despite ignoring the effects of the interfering FCs, regardless, whether the UFR, hard-FFR and soft-FFR scenarios are considered. It is clearly shown that as expected, the received SIR of the hard-FFR scenario is better than that of the soft-FFR and UFR scenario, which is due to the fact that no CCI is imposed either by the tier-one MBSs or by the DAEs within the tier-one MCs, while the received SIR of the UFR scenario becomes the worst amongst all frequency reuse patterns, as a result of the strong CCI within the same MC.

When the mediocre-density femtocellular scenario of Fig. 3 is considered in conjuction with $N_{f}=50$, the simulation 


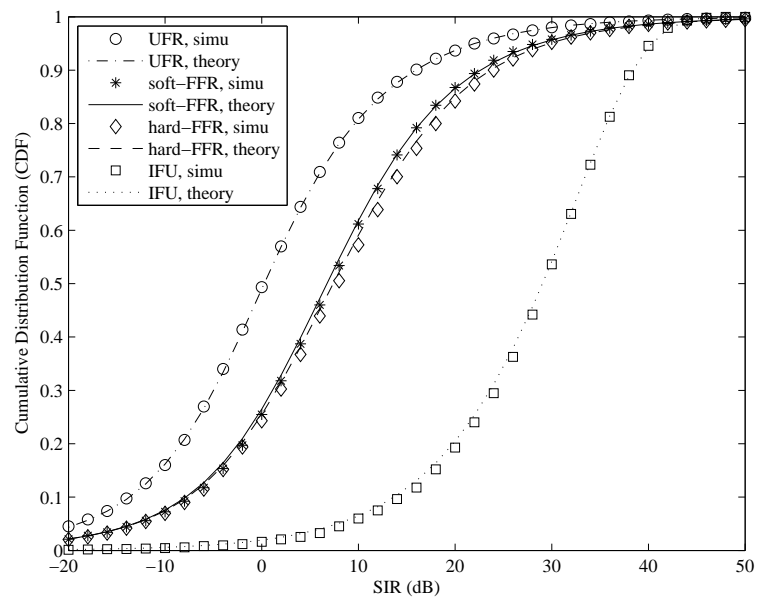

Fig. 3: The CDF of the received SIR, when the number of femtocells within each macrocell is $N_{f}=50$.

results become slightly better than the theoretical results, because we only considered the CCI arriving from the interfering FBSs within a circle having a radius of $R_{m}$, but ignored the CCI imposed by the FBSs far away from the user considered in our simulations. Fig. 3 also shows the CDF of the spatially averaged received SIR for the worst-case IFUs, which are located at the cell-edge of their serving FBSs. Our simulation results demonstrate that the OP of the worst-case IFUs remains quite low, even if the SIR threshold $\gamma_{t h}$ is set to be as high as $20 \mathrm{~dB}$, because the CCI emanating from the neighboring FBSs is significantly reduced owing to the attenuation of walls.

\section{B. Optimised design for DAS-aided twin-layer cellular net- works}

In order to highlight the importance of optimal parameter design, we compare the performance of both the OP-based design and of the throughput-based design of our DAS-aided twin-layer network, where a QoS constraint of $\eta=0.2$ is considered. We also considered a static system configuration of $r_{d}=\frac{2}{3}$ and $\mu=1.0$ as the benchmarker, which is referred to as System 1 (S1).

1) OP-based design: Fig. 4 and Fig. 5 illustrate the optimised system configurations as a function of the number of FCs $N_{f}$ in the UFR, hard-FFR and soft-FFR scenarios. Observe from both figures that the UFR scenario relies on the near-constant parameters of $r_{d} \approx 0.62$ and $\mu \approx 1$, which are independent of the number of FCs $N_{f}$. In other words, the system parameters of the UFR scenario can be configured separately, entirely without considering the impact of the interfering FCs. However, the optimised radius $r_{d}$ of the DAS tends to decrease upon increasing the number of FCs for both the hard-FFR and soft-FFR scenario, which are sensitive to the density of the FCs. Furthermore, the optimised power allocation factor $\mu$ tends to increase, as the number of FCs $N_{f}$ increases. In the CCR, the performance of both the hardFFR and of the soft-FFR scenario becomes worse than that of the CER, when the density of FCs increases. As a result, the

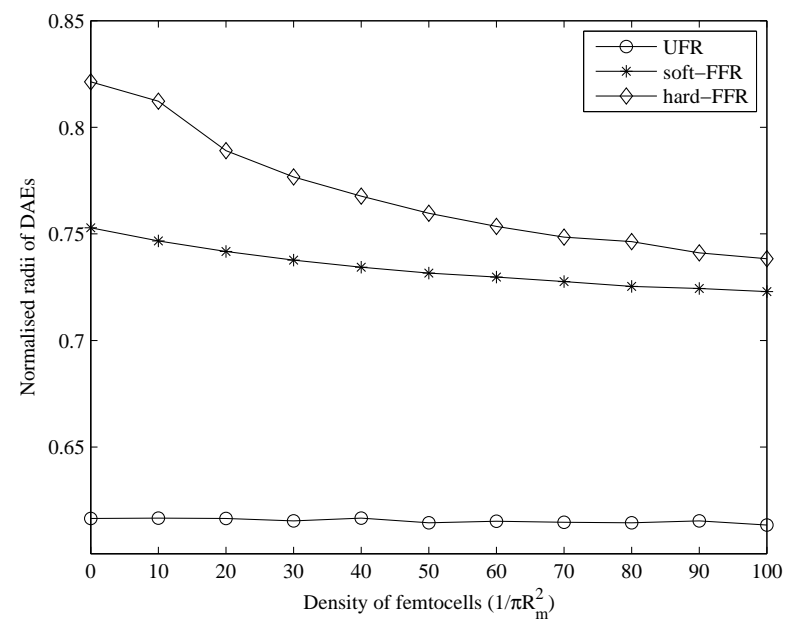

Fig. 4: The optimised normalised radii of DAS $r_{d}$ in the OPbased design.

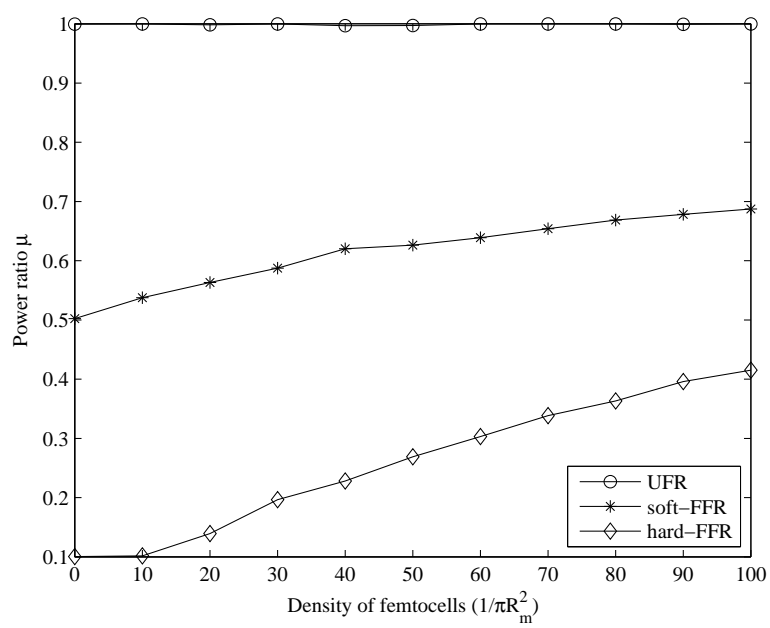

Fig. 5: The optimised power allocation $\mu$ in the OP-based design.

OMUs have to be handed over from the MBS to the DAEs of the CER of twin-layer networks in the presence of high-density interfering FCs, which may be viewed, as the MCs being split into several DAS-based small cells in order to maintain a low OP.

Fig. 6 compares the spatially averaged OP of S1 and of the GA-aided near-optimal networks, where the SIR threshold is set to $\gamma_{t h}=3 d B$. It is clearly shown in Fig. 6 that as expected, the spatially averaged OP increases with the number of FCs within all scenarios considered. More specifically, the OP of the UFR scenario is significantly higher than that of both the hard-FFR and soft-FFR scenario, duo to the fact that the CCI imposed on the OMUs of the UFR scenario is significantly higher than in the other two scenarios. Furthermore, the OP of the hard-FFR scenario is lower than that of the softFFR, which is achieved at the cost of a reduced throughput. However, the OP of the hard-FFR increases more rapidly 


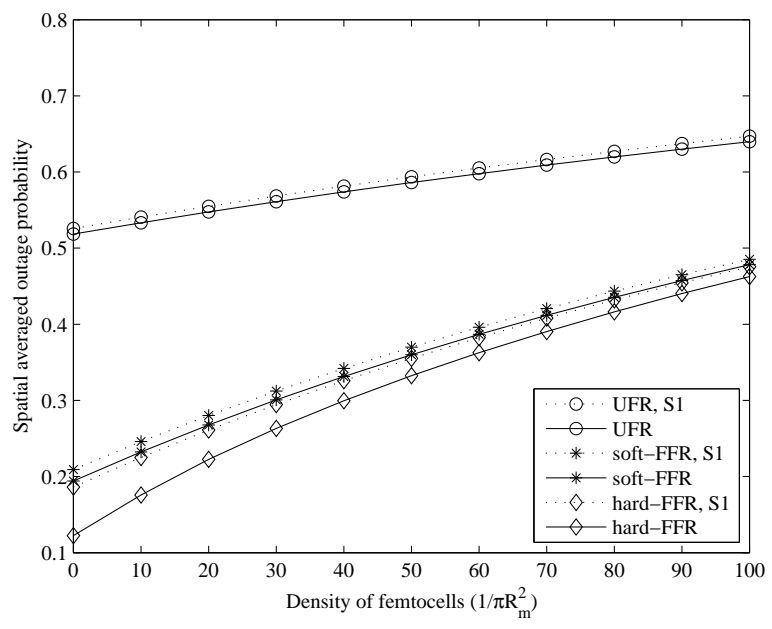

Fig. 6: The spatially averaged OP in the OP-based design, when the SIR threshold is $\gamma_{t h}=3 d B$.

than that of soft-FFR and then becomes lower than that of the soft-FFR scenario, when the density of FCs $\lambda$ is high. Fig. 6 also shows that the OP of the GA-based near-optimal networks always remains better than that of the benchmarker $\mathrm{S} 1$, especially for the low-density femtocellular hard-FFR scenario.

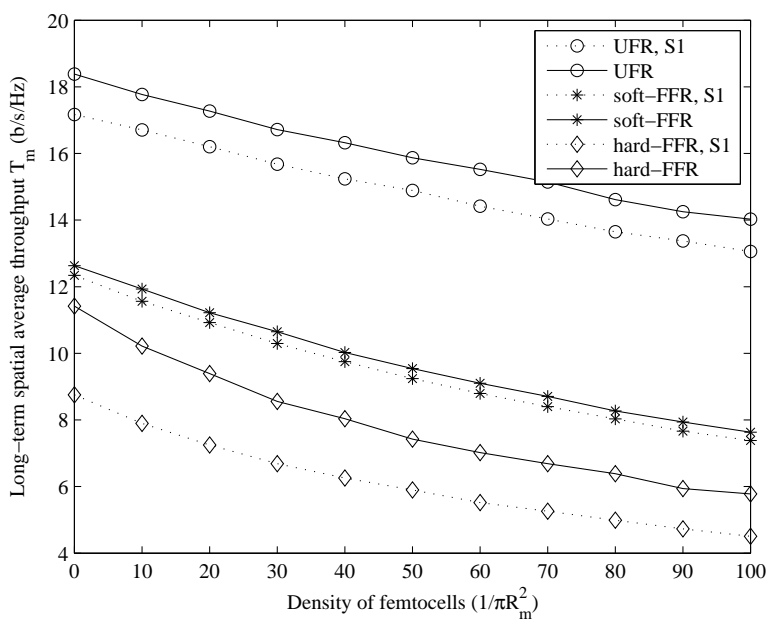

Fig. 7: The long-term spatially averaged macrocell throughput in throughput-based design.

2) Throughput-based design: Fig. 7 illustrates the achievable spatially averaged MC throughput of both the benchmarker S1 and of the GA-based near-optimal networks using our throughput-based design, when the UFR, hard-FFR or softFFR frequency reuse pattern is employed. The achievable MC throughput of the optimised design decreases, as the number of interfering FCs $N_{f}$ increases. This is because the CCI imposed by high-density FBSs is stronger than that of lowdensity systems. Due to the aggressive frequency reuse of the UFR scenario, the attainable MC throughput is much higher than that of the FFR scenarios, albeit this is achieved at the cost of a high OP. In other words, a trade-off between the attainable throughout and the tolerable OP has to be struck. Furthermore, Fig. 7also illustrates that the achievable throughput of the GAbased near-optimal networks is higher than that of the static configuration S1, and a larger gap is observed for the hardFFR scenario, which is more sensitive to the specific choice of the system parameters.

\section{Outage probability of worst-case IFUs under optimal UFR scenario}

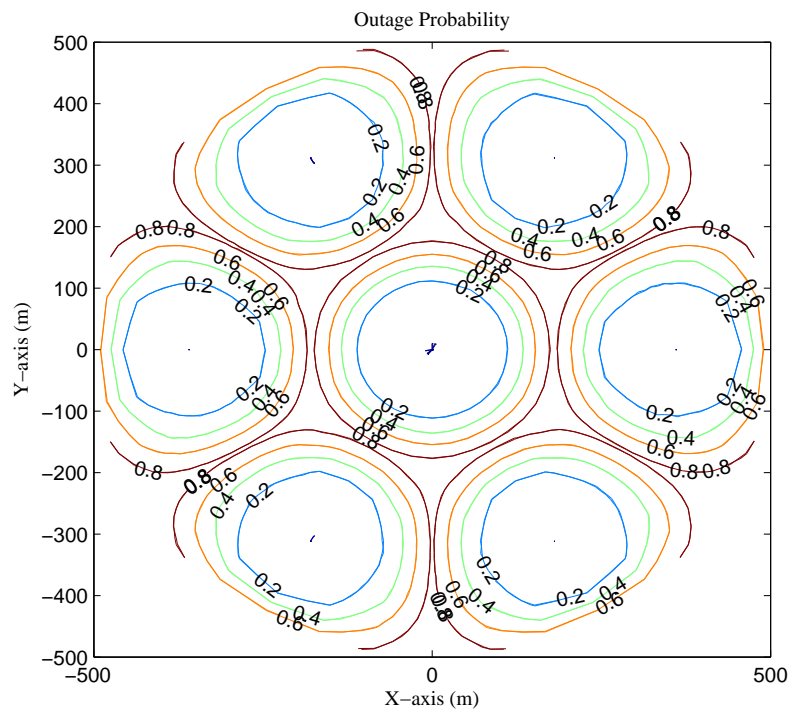

Fig. 8: The OP of OMU for mediocre-density femtocellular networks, when the number of femtocells is $N_{f}=50$ and $\gamma_{t h}=3 d B$.

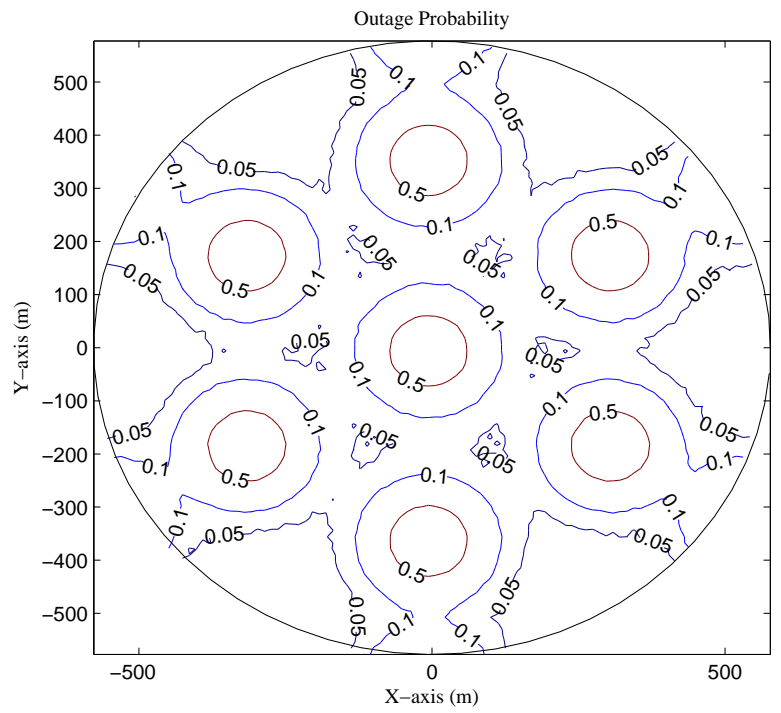

Fig. 9: The OP of worst-case IFUs for mediocre-density femtocellular networks, when the number of femtocells is $N_{f}=50$ and $\gamma_{t h}=20 \mathrm{~dB}$. 
Let us now investigate the OP of OMUs and IFUs in the context of twin-layer cellular networks associated with UFR, where the average number of FCs in each MC is $N_{f}=50$. It is shown in Fig. 8 that the outage of the OMUs is lower than $20 \%$, when they are within $100 m$ from their serving MBSs. However, the OP of OMUs becomes higher, when they are roaming far away from their serving MBSs, because we have a weaker desired signal and a stronger interference emanating from the neighbouring DAEs. Observe from Fig. 9 that the OP $\mathcal{O}_{f}\left(\gamma_{t h}\right)$ of the worst-case IFU is lower than $5 \%$, when its serving FBS is far from both the MBSs and from the DAEs. However, the OP of IFUs becomes higher than $50 \%$, when the serving FBSs are located in the vicinity of either the MBS or of the DAEs. As a result, an outage is likely to occur with a high probability and the FCs may have to be allocated to orthogonal frequency bands wrt that of the MC.

\section{CONCLUSIONS}

In this paper, we have provided the analysis and the GAbased near-optimal design of DAS-aided twin-layer cellular networks associated with UFR, hard-FFR and soft-FFR scenarios, where both the per-layer OP as well as the longterm spatially averaged throughput were derived. The GAbased near-optimal design of this twin-layer cellular system for the UFR, hard-FFR as well as soft-FFR scenarios has been investigated, with the goal of minimising the OP or maximising the spatially averaged throughput. We have found that the optimised design for the UFR scenario is independent of the density of the interfering FCs, suggesting that the system can be optimised without considering the impact of the FCs. As for the hard-FFR and the soft-FFR scenario operating in the presence of high-density FCs, the optimised MC tends to migrate to smaller cells illuminated by the DAEs in order to maintain a low OP. Our received SIR results of the worstcase IFUs also demonstrated that an outage near the MBSs or the DAEs is likely to occur with a high probability. Hence in this scenario the FCs should be allocated orthogonal frequency bands.

\section{APPENDIX A}

\section{OUTAGE PROBABILITY OF OMUS WITHIN UFR SCENARIO}

When UFR is considered, the received SIR $\gamma_{u}$ of OMU $u$ within the CCR is rewritten from Eq. (1) as

$$
\gamma_{u}=\frac{h_{0}}{I_{1}+I_{2}+I_{3}}
$$

where we have $I_{1}=\frac{1}{d_{0}^{-\alpha_{1}}} \sum_{i \neq 0} d_{i}^{-\alpha_{1}} h_{i}, \quad I_{2}=$ $\frac{P_{a}}{P_{b} d_{0}^{-\alpha_{1}}} \sum_{i} \sum_{j} d_{i, j}^{-\alpha_{1}} h_{i, j}$ and $I_{3}=\frac{P_{f}}{P_{b} d_{0}^{-\alpha_{1}} W} \sum_{n} d_{f_{n}}^{-\alpha_{1}} g_{n}$. Due to the fact that the interference terms $I_{1}, I_{2}$ and $I_{3}$ are independent of each other and the channel gain $h_{i}$ is negative exponentially decaying, the OP $\mathcal{O}_{u}\left(\gamma_{t h} \mid r_{u}, \theta_{u}\right)$ of the CCR's
OMU at a radius of $r_{u}$ and angle of $\theta_{u}$ may be written as

$$
\begin{aligned}
\mathcal{O}_{u}\left(\gamma_{t h} \mid r_{u}, \theta_{u}\right) & =1-P\left(\gamma_{u} \geq \gamma_{t h} \mid r_{u}, \theta_{u}\right) \\
& =1-P\left[h_{0} \geq \gamma_{t h}\left(I_{1}+I_{2}+I_{3}\right) \mid r_{u}, \theta_{u}\right] \\
& =1-E_{I_{1}, I_{2}, I_{3}}\left\{e^{\left[-\gamma_{t h}\left(I_{1}+I_{2}+I_{3}\right)\right]}\right\} \\
& =1-E_{I_{1}, I_{2}, I_{3}}\left\{e^{\left(-\gamma_{t h} I_{1}\right)} e^{\left(-\gamma_{t h} I_{2}\right)} e^{\left(-\gamma_{t h} I_{3}\right)}\right\} \\
& =1-\mathcal{L}_{I_{1}}\left(\gamma_{t h}\right) \mathcal{L}_{I_{2}}\left(\gamma_{t h}\right) \mathcal{L}_{I_{3}}\left(\gamma_{t h}\right),
\end{aligned}
$$

where $\mathcal{L}_{I_{1}}, \mathcal{L}_{I_{2}}$ and $\mathcal{L}_{I_{3}}$ denote the Laplace transform of the random variable $I_{1}, I_{2}$ and $I_{3}$, respectively. The term $I_{1}$ is the linear sum of independent exponential distributed random variable $h_{i}$, the Laplace transform of $\mathcal{L}_{I_{1}}$ can be directly derived as

$$
\begin{aligned}
\mathcal{L}_{I_{1}}\left(\gamma_{t h}\right) & =E\left[e^{\left(-\frac{\gamma_{t h}}{d_{0}^{-\alpha_{1}} \sum_{i \neq 0} d_{i}^{-\alpha_{1}} h_{i}}\right)}\right] \\
& =\prod_{i \neq 0} E\left[e^{\left(-\frac{\gamma_{t h} d_{i}^{-\alpha_{1}}}{d_{0}^{-\alpha_{1}}} h_{i}\right)}\right] \\
& \stackrel{(a)}{=} \prod_{i \neq 0}\left(1+\frac{\gamma_{t h} d_{i}^{-\alpha_{1}}}{\left.d_{0}^{-\alpha_{1}}\right)^{-1}},\right.
\end{aligned}
$$

where $(a)$ follows the Laplace transform of exponential random variables. Similarly, the Laplace transform of the interference term $I_{2}$ may be written as

$$
\mathcal{L}_{I_{2}}\left(\gamma_{t h}\right)=\prod_{i} \prod_{j}\left(1+\frac{P_{a} d_{i, j}^{-\alpha_{1}}}{P_{b} d_{0}^{-\alpha_{1}}} \gamma_{t h}\right)^{-1}
$$

The Laplace transform of the sum of SPPP $I_{3}$ is given by

$$
\begin{aligned}
& \mathcal{L}_{I_{3}}\left(\gamma_{t h}\right)=E_{\Psi, g_{n}}\left[e^{\left(-\sum_{n \in \Psi} \frac{P_{f} \gamma_{t h}}{P_{b} d_{0}^{-\alpha_{1} W} d_{f_{n}}^{-\alpha_{1}} g_{n}}\right)}\right] \\
& =E_{\Psi, g_{n}}\left[\prod_{n \in \Psi} e^{\left(-\frac{P_{f} \gamma_{t h}}{P_{b} d_{0}^{-\alpha_{1}} W} d_{f_{n}}^{-\alpha_{1}} g_{n}\right)}\right] \\
& =E_{\Psi}\left\{\prod_{n \in \Psi} E_{g_{n}}\left[e^{\left(-\frac{P_{f} \gamma_{t h}}{P_{b} d_{0}^{-\alpha_{1}} W} d_{f_{n}}^{-\alpha_{1}} g_{n}\right)}\right]\right\} \\
& \stackrel{(b)}{=} E_{\Psi}\left[\prod_{n \in \Psi}\left(1+\frac{P_{f} \gamma_{t h}}{P_{b} d_{0}^{-\alpha_{1}} W} d_{f_{n}}^{-\alpha_{1}}\right)^{-1}\right] \\
& \stackrel{(c)}{=} e^{\left[-2 \pi \lambda \int_{0}^{\infty}\left(1-\frac{1}{1+\frac{P_{f} \gamma_{t h}}{P_{b} d_{0}^{-\alpha_{1}} W} t^{-\alpha_{1}}}\right) t d t\right]} \\
& =e^{\left[-\pi \lambda C_{\alpha_{1}}\left(\frac{P_{f} \gamma_{t h}}{P_{b} d_{0}^{-\alpha_{1}} W}\right)^{\frac{2}{\alpha_{1}}}\right]},
\end{aligned}
$$

where $(b)$, again, follows the Laplace transform of exponential distributed variables and $(c)$ follows from the Probability Generating Functional (PGFL) of the SPPP [27]. Substituting Eq. (23), Eq. (24) and Eq. (25) into Eq. (22), the OP of OMU within the CCR at a radius of $r_{u}$ and angle of $\theta_{u}$ can be 
derived as

$$
\mathcal{O}_{u}\left(\gamma_{t h} \mid r_{u}, \theta_{u}\right)=1-e^{\left[-\pi \lambda C_{\alpha_{1}}\left(\frac{P_{f} \gamma_{t h}}{P_{b} d_{0}^{-\alpha_{1} W}}\right)^{\frac{2}{\alpha_{1}}}\right]} \mathcal{H}_{1} .
$$

Following a similar derivation for the OP of the CCR's OMUs, the OP of the CER's OMU at a radius of $r_{u}$ and angle of $\theta_{u}$ can be expressed as

$$
\mathcal{O}_{u}\left(\gamma_{t h} \mid r_{u}, \theta_{u}\right)=1-e^{\left[-\pi \lambda C_{\alpha_{1}}\left(\frac{P_{f} \gamma_{t h}}{P_{a} d_{0, k}^{-\alpha_{1} W}}\right)^{\frac{2}{\alpha_{1}}}\right]} \mathcal{H}_{2} .
$$

\section{APPENDIX B}

\section{OUTAGE PROBABILITY OF IFUS WITHIN UFR SCENARIO}

We will then consider the worst-case OP of IFUs having a distance of $R_{f}$ from the serving FBS, which is located at a radius of $r_{f_{n}}$ and angle of $\theta_{f_{n}}$ far from the home MBS. Similarly, the SIR $\gamma_{u}$ of Eq. (5) is rewritten as

$$
\gamma_{u}=\frac{g_{n}}{I_{4}+I_{5}+I_{6}}
$$

where we have $I_{4}=\sum_{i} \frac{P_{b} d_{i}^{-\alpha_{1}}}{P_{f} W R_{f}^{-\alpha_{2}}} h_{i}, I_{5}=$ $\sum_{i} \sum_{j} \frac{P_{a} d_{i}^{-\alpha_{1}}}{P_{f} W R_{f}^{-\alpha_{2}}} h_{i, j}$ and $I_{6}=\sum_{j \neq n} \frac{d_{f_{j}}^{-\alpha_{1}}}{W^{2} R_{f}^{-\alpha_{2}}} g_{j}$. Again, the OP of the IFUs located at the cell-edge of FBS $B_{n}^{f}$, which has a radius of $r_{f_{n}}$ and angle of $\theta_{f_{n}}$ from the home MBS, is given as

$$
\mathcal{O}_{u}\left(\gamma_{t h} \mid r_{f_{n}}, \theta_{f_{n}}\right)=1-\mathcal{L}_{I_{4}}\left(\gamma_{t h}\right) \mathcal{L}_{I_{5}}\left(\gamma_{t h}\right) \mathcal{L}_{I_{6}}\left(\gamma_{t h}\right),
$$

where $\mathcal{L}_{I_{4}}\left(\gamma_{t h}\right), \mathcal{L}_{I_{5}}\left(\gamma_{t h}\right)$ and $\mathcal{L}_{I_{6}}\left(\gamma_{t h}\right)$ are derived similarly from Eq. 23) and Eq. 25, yielding

$$
\begin{aligned}
& \mathcal{L}_{I_{4}}\left(\gamma_{t h}\right)=\prod_{i}\left(1+\frac{P_{b} \gamma_{t h} d_{i}^{-\alpha_{1}}}{P_{f} W R_{f}^{-\alpha_{2}}}\right)^{-1} \\
& \mathcal{L}_{I_{5}}\left(\gamma_{t h}\right)=\prod_{i} \prod_{j}\left(1+\frac{P_{a} \gamma_{t h} d_{i, j}^{-\alpha_{1}}}{P_{f} W R_{f}^{-\alpha_{2}}}\right)^{-1} \\
& \mathcal{L}_{I_{6}}\left(\gamma_{t h}\right)=e^{\left[-\pi \lambda C_{\alpha_{1}}\left(\frac{\gamma_{t h}}{W^{2} R_{f}^{-\alpha_{2}}}\right)^{\frac{2}{\alpha_{1}}}\right]} .
\end{aligned}
$$

Substituting Eq. (30), Eq. (31) and Eq. (32) into the Eq. (29), we arrive at Lemma 2.

\section{APPENDIX C}

\section{The Optimis Ation Process of Genetic Algorithms}

1) Initialisation: A population of solutions is randomly generated, which is composed of $N_{\text {ind }}$ number of $L$-bit (candidate solutions) chromosomes and each chromosome represents an individual solution $\omega$. In this paper, we used $N_{\text {ind }}=20$ and $L=20$.

2) Evaluate the fitness $F\left(\omega_{i}\right)$ of each chromosome $\omega_{i}$ in the population, where the individual's fitness $F\left(\omega_{i}\right)$ is computed either as the individual's OP $\mathcal{O}_{m}$ or the MC throughput $T_{m}$, which is formulated as

$$
\begin{aligned}
& \mathcal{O}_{m}\left(\omega_{i}\right)=\int_{0}^{R_{m}} \int_{0}^{2 \pi} \frac{r}{\pi R_{m}^{2}} \mathcal{O}_{u}\left(\gamma_{t h} \mid r_{u}=r, \theta_{u}=\theta\right) d r d \theta \\
& T_{m}\left(\omega_{i}\right)=\rho_{i} T_{m_{1}}\left(\omega_{i}\right)+\rho_{o} N_{a} T_{m_{2}}\left(\omega_{i}\right),
\end{aligned}
$$

where $\mathcal{O}_{m}\left(\gamma_{t h} \mid r, \theta\right)$ is a function of the candidate solution $\omega_{i}$.

3) Generate the new population with the aid of using the standard recombination and mutation operation of the GA [29]:

a) Crossover: Select multiple pairs of "parent" individuals from the population for breeding the "offspring". For each pair of "parent", arrange for the cross-over operation at a randomly chosen point with probability $P_{c}$, which is set to $P_{c}=0.7$ in this paper.

b) Mutation: Alter the gene value in each chromosome of the selected individuals with a probability of $P_{m}$. We used a low mutation probability of $P_{m}=0.05$ for avoiding primitive random search.

4) Go to step 2 and continue the iteration, until the number of generations reaches its maximum value of $N_{\max }=$ 50 .

\section{REFERENCES}

[1] A. Ghosh, N. Mangalvedhe, R. Ratasuk, B. Mondal, M. Cudak, E. Visotsky, T. A. Thomas, J. G. Andrews, P. Xia, H. S. Jo, H. S. Dhillon, and T. D. Novlan, "Heterogeneous cellular networks: From theory to practice," IEEE Communications Magazine, vol. 50, pp. 54-64, Jun. 2012.

[2] J. Zhang and J. G. Andrews, "Distributed antenna systems with randomness," IEEE Transactions on Wireless Communications, vol. 7, pp. 36363646, Sep. 2008.

[3] X. You, D. Wang, P. Zhu, and B. Sheng, "Cell edge performance of cellular mobile systems," IEEE Journal on Selected Areas in Communications, vol. 29, pp. 1139-1150, Jun. 2011.

[4] R1-104368, "Summary of the description of candidate eicic solutions," tech. rep., 3GPP Std., Madrid, Aug. 2010.

[5] R1-104661, "Comparison of time-domain eicic solutions," tech. rep., 3GPP Std., Madrid, Aug. 2010.

[6] R. Steele and L. Hanzo, Mobile Radio Communications. John WileyIEEE Press, 1999.

[7] X. Xu, R. Zhang, S. Ghafoor, and L. Hanzo, "Imperfecr digitalfiber-optic-link-based cooperative distributed antennas with fractional frequency reuse in multicell multiuser networks," IEEE Transcations on Vehicular Techonology, vol. 60, pp. 4439-4449, Oct. 2011.

[8] G. Boudreau, J. Panicker, N. Guo, R. Wang, and S. Vrzic, "Interference coordination and cancellation for $4 \mathrm{G}$ networks," IEEE Communications Magazine, vol. 47, pp. 74-81, Apr. 2009.

[9] L. Chen and D. Yuan, "Generalized frequency reuse schemes for OFDMA networks: Optimization and comparison," in Proc. IEEE VTC, pp. 1-5, May 2008.

[10] T. D. Novlan, R. K. Ganti, A. Ghosh, and J. G. Andrews, "Analytical evaluation of fractional frequency reuse for OFDMA cellular networks," IEEE Transactions on Wireless Communications, vol. 10, pp. 42944305, Dec. 2011.

[11] H. Lei, L. Zhang, X. Zhang, and D. Yang, "A novel multi-cell OFDMA system structure using fractional frequency reuse," in Proc. IEEE PIMRC, pp. 1-5, Sep. 2007.

[12] H. Fujii and H. Yoshino, "Theoretical capacity and outage rate of OFDMA cellular system with fractional frequency reuse," in Proc. IEEE VTC, pp. 1676-1680, May 2008.

[13] T. Novlan, J. G. Andrews, I. Sohn, and R. K. Ganti, "Comparison of fractional frequency reuse approaches in the OFDMA cellular downlink," in Proc. IEEE GLOBECOM, pp. 1-5, Jan. 2010.

[14] Z. Xu, G. Y. Li, and C. Yang, "Optimal threshold design for FFR schemes in multi-cell OFDMA networks," in Proc. IEEE ICC, pp. 1-5, Jul. 2011.

[15] M. Assaad, "Optimal fractional frequency reuse FFR in multicellular OFDMA system," in Proc. IEEE VTC, pp. 1-5, Sep. 2008.

[16] A. Alsawah and I. Fijalkow, "Optimal frequency-reuse partitioning for ubiquitous coverage in cellular systems," in Proc. European Signal Processing Conference, pp. 1-5, Aug. 2008. 
[17] S. H. Ali and V. C. M. Leung, "Dynamic frequency allocation in fractional frequency reused OFDMA networks," IEEE Transactions on Wireless Communications, vol. 8, pp. 4286-4295, Aug. 2009.

[18] V. Chandrasekhar and J. G. Andrews, "Femtocell networks: A survey," IEEE Communications Magazine, vol. 46, pp. 59-67, Sep. 2008.

[19] V. Chandrasekhar and J. G. Andrews, "Uplink capacity and interference avoidance for two-tier femtocell networks," IEEE Transactions on Wireless Communications, vol. 8, pp. 3498-3509, July 2009.

[20] G. Jeney, "Practical limits of femtocells in a realistic environment," in Proc. IEEE VTC, pp. 1-5, May 2011.

[21] V. Chandrasekhar, M. Kountouris, and J. G. Andrews, "Coverage in multi-antenna two-tier networks," IEEE Transactions on Wireless Communications, vol. 8, pp. 5314-5327, Oct. 2009.

[22] V. Chandrasekhar and J. G. Andrews, "Spectrum allocation in tiered cellular networks," IEEE Transactions on Information Theory, vol. 57, pp. 3059-3068, Oct. 2009.

[23] K. Yeung and S. Nanda, "Channel management in microcell/macrocell cellular radio systems," IEEE Transactions on Vehicular Technology, vol. 45, pp. 601-612, Nov. 1996.

[24] J. Yun and K. G. Shin, "Adaptive interference management of OFDMA femtocells for co-channel deployment," IEEE Journal on Selected Areas in Communications, vol. 29, pp. 1225-1241, Jun. 2011.

[25] Y. Liang, W. Chung, G. Ni, and I. Chen, "Resource allocation with interference avoidance in ofdma femtocell networks," IEEE Transcations on Vehicular Techonology, vol. 61, pp. 2243-2255, Jun. 2012.

[26] D. L. Perez, A. Ladanyi, A. Juttner, and J. Zhang, "OFDMA femtocells: A self-organizing approach for frequency assignment," in Proc. IEEE PIMRC, pp. 2202-2207, Sep. 2009.

[27] M. Haenggi, J. G. Andrews, F. Baccelli, O. Dousse, and M. Franceschetti, "Stochastic geometry and random graphs for the analysis and design of wireless networks," IEEE Journal on Selected Areas in Communications, vol. 27, pp. 1029-1046, Sep. 2009.

[28] F. Baccelli, B. Blaszczyszyn, and P. Muhlethaler, "Stochastic analysis of spatial and opportunistic aloha," IEEE Journal on Selected Areas in Communications, vol. 27, pp. 1105-1119, Sep. 2009.

[29] D. E. Goldberg, Genetic Algorithms in Search, Optimization and Machine Learning. Addison Wesley Publishing Company, 1989.

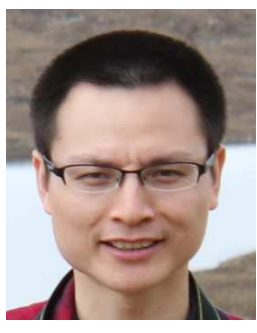

Jie Zhang received his B.Eng. degree, M. Eng. degree and Ph.D. degree, in 2005, 2008 and 2013, respectively, from the University of Electronic Science and Technology of China (UESTC), Chengdu, China. From September 2010 to September 2012, he has been a Visiting Student with the Communications Research Group, School of Electronics and Computer Science, University of Southampton, Southampton, UK. Currently, he is with the Sichuan Electronic Power Research Institute, State Grid Coorperation of China. His research interests include cooperative communications, cellular network optimisation as well as high-speed backbone network.

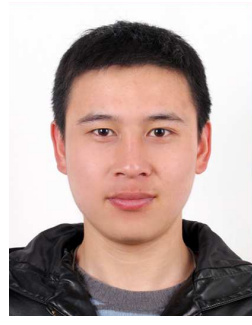

Fan Jin received the B.Eng. and M.Sc. degrees in electronics and information engineering from Huazhong University of Science and Technology (HUST), Wuhan, China, in 2008 and 2010, respectively. He is currently working towards the Ph.D. degree in the Communications Group, School of Electronics and Computer Science, University of Southampton, Southampton, UK. He has received a scholarship under the UK-China Scholarships for Excellence Programme. His research interests include multi-user communications, radio resource allocation, spectrum sensing and interference management in femtocells and heterogeneous networks.

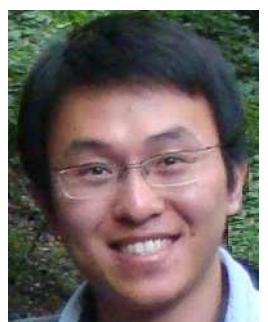

Rong Zhang received his $\mathrm{PhD}$ (Jun 09) from Southampton University, UK and his BSc (Jun 03) from Southeast University, China. Before doctorate, he was an engineer (Aug 03-July 04) at China Telecom and a research assistant (Jan 06-May 09) at Mobile Virtual Center of Excellence (MVCE), UK. After being a post-doctoral researcher (Aug 09-July 12) at Southampton University, he took industrial consulting leave (Aug 12-Jan 13) for Huawei Sweden R\&D as a system algorithms specialist. Since Feb 13, he has been appointed as a lecturer at CSPC group of ECS, Southampton University. He has $25+$ journals in prestigious publication avenues (e.g. IEEE, OSA) and many more in major conference proceedings. He regularly serves as reviewer for IEEE transactions/journals and has been several times as TPC member/invited session chair of major conferences. He is a member of IEEE and the recipient of joint funding of MVCE and EPSRC. He is also a visiting researcher of Nanjing University under Worldwide University Network (WUN).

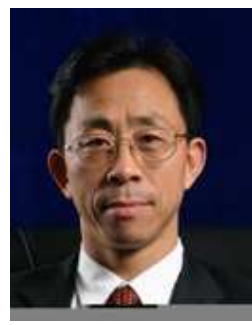

Guangjun Li(M'07) received the M.S. degree from the University of Electronic Science and Technology of China (UESTC), Chengdu, China, in 1985. Since 1985 he has been with the School of Communication and Information Engineering, UESTC. From 1991 to 1992, he was a visiting scholar with RWTH Aachen University, Germany. He currently holds the Chair of Communication Integrated Circuits and Systems Engineering Center of UESTC and the leader of the Wireless Communications and ASIC Laboratory, working on a range of research projects in the field of communication systems sponsored by National Natural Science Foundation of China, National 863 High Technology Development Project and National ASIC Chip Design Project, China. He has published various papers in the area of communication systems, wireless communication networks, embedded systems, application-specific integrated circuits and silicon-on-chop design.

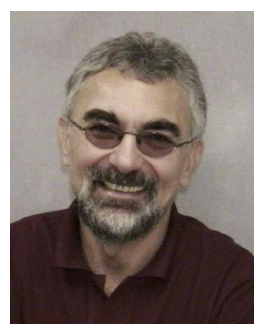

Lajos Hanzo FREng, FIEEE, FIET, Fellow of EURASIP, DSc received his degree in electronics in 1976 and his doctorate in 1983. In 2009 he was awarded the honorary doctorate "Doctor Honoris Causa" by the Technical University of Budapest. During his 37-year career in telecommunications he has held various research and academic posts in Hungary, Germany and the UK. Since 1986 he has been with the School of Electronics and Computer Science, University of Southampton, UK, where he holds the chair in telecommunications. He has successfully supervised $80+\mathrm{PhD}$ students, co-authored 20 John Wiley/IEEE Press books on mobile radio communications totalling in excess of 10000 pages, published 1300+ research entries at IEEE Xplore, acted both as TPC and General Chair of IEEE conferences, presented keynote lectures and has been awarded a number of distinctions. Currently he is directing a 100-strong academic research team, working on a range of research projects in the field of wireless multimedia communications sponsored by industry, the Engineering and Physical Sciences Research Council (EPSRC) UK, the European Research Council's Advanced Fellow Grant and the Royal Society's Wolfson Research Merit Award. He is an enthusiastic supporter of industrial and academic liaison and he offers a range of industrial courses. He is also a Governor of the IEEE VTS. During 2008 - 2012 he was the Editor-in-Chief of the IEEE Press and a Chaired Professor also at Tsinghua University, Beijing. His research is funded by the European Research Council's Senior Research Fellow Grant. For further information on research in progress and associated publications please refer to http://www-mobile.ecs.soton.ac.uk Lajos has 17 000+ citations. 